\title{
ACTIVE MODELS FOR DIGITALLY ENABLED CREATIVE BUSINESS NETWORKS
}

\author{
John Krogstie ${ }^{1}$, Håvard D. Jørgensen ${ }^{1}$ and Frank Lillehagen ${ }^{2}$

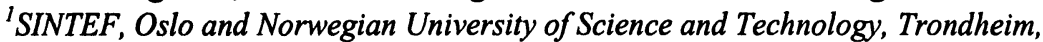 \\ Norway\{jok,hdj\}@informatics.sintef.no \\ ${ }^{2}$ Computas, Norwayfli@computas.com
}

\begin{abstract}
Co-operation across organisational boundaries is increasing. When such cooperation moves beyond the trade of well-defined products, there is a need for a flexible infrastructure that supports not only information exchange, but also knowledge creation, sharing, utilisation and learning. Active models constitute a general technique for increasing the flexibility of computerised information systems. Such models are available for manipulation by the users at runtime, and the model contents influence the behaviour of the system. By altering the models, the users can thus tailor the system to fit their particular needs and preferences. This paper explores the use of active models to support digitally enabled creative business networks. In particular, we look at how enterprise and work process models can support virtual enterprise formation, engineering, operation, and learning. Our initial results indicate the great potential of active models in this area.
\end{abstract}

Key words: eBusiness, eWork, Enterprise Modelling, Active models

The original version of this chapter was revised: The copyright line was incorrect. This has been corrected. The Erratum to this chapter is available at DOI: 10.1007/978-0-387-35604-4_20 


\section{INTRODUCTION}

Increasingly, outsourcing, electronic business and globalisation are enabled by the Internet and other information systems (IS). Most of the future growth is expected in the service sector, and while computerisation automates routine procedures, how to support knowledge-based co-operation remains a challenge. In knowledge-based virtual enterprises, each partner company contributes unique and complimentary competence vital for the success of the joint enterprise. The situated, contingent, and emergent nature of work [26] similarly requires flexible support infrastructures. Flexibility is seldom offered by the tools currently available for interorganisational cooperation, like e-business frameworks [25], workflow management systems $[1,4,24,28]$, enterprise resource planning systems (ERP) $[1,20]$ etc. From past experience with developing flexible groupware and workflow systems $[4,13-15,22]$, we have refined an active model approach to flexible information systems $[5,12,16]$. Active models are available to the users at runtime, and the behaviour of the information system is partially controlled by the models. By altering the models the users can thus modify the behaviour of the system to fit their current situation. This paper explores the use of active models to support knowledge intensive interorganisational cooperation, based on the intermediate results of the ongoing EXTERNAL project [7].

\subsection{The structure of this paper}

The following section looks at how knowledge intensive projects are performed in digitally enabled creative business networks. Challenges regarding flexible organisation, effective communication, learning and knowledge sharing are outlined. We then describe our approach of active models, and illustrate how this approach can help solve the challenges identified. The validation has three parts. First, a brief description of the current implementation of the IS infrastructure of the EXTERNAL project is given, showing that the approach is technically feasible. Second, the ongoing and planned use of this infrastructure in industrial case studies help us assess social and practical concerns of representative organisations. We will here also present our ongoing approach to evaluate the results of introducing the new infrastructure to support the cases. And third, a brief comparison with other co-operative information systems approaches highlights both the unique aspects of our approach and an emerging trend towards more active use of models independent of our efforts. Finally some directions for further research are offered. 


\section{DIGITALLY ENABLED CREATIVE BUSINESS NETWORKS}

In the EXTERNAL (Extended Enterprise Resources, Networks, and Learning) project [7], our aim is to facilitate inter-organisational cooperation in knowledge intensive tasks. To investigate this, users and researchers have captured requirements from three different virtual enterprises. Based on these requirements we have implemented an IS infrastructure that integrates and further extended application services previously developed by the EXTERNAL partners, and defined assessment criteria for the practical use of the infrastructure in the three enterprises. The main challenges of digitally enabled creative business networks that this analysis has identified, include:

- Support for communication, building a common understanding of the terminology, tasks, resources, results, competence and skills that are needed to reach the goals of the joint enterprise.

- Support for rapid formation of virtual organisations, and for allowing partners to be included or to leave the co-operation as the enterprise progresses.

- Different organisations have heterogeneous IS infrastructures, today often bridged only by email and publish-oriented portals, not by work support environments.

- The planning of an interorganisational enterprise is a joint effort, but at the same time some partners may wish to keep private certain details of how they do their internal work. This calls for sophisticated and flexible access control policies.

- Different organisations have different quality control procedures with potentially conflicting standards.

- The network as a whole, and individual partners, must be able to learn and improve from their common experience, and to reuse the knowledge developed there in future enterprises.

- We must be able to integrate capabilities from the different organisations, to form effective teams across organisational boundaries and local cultures.

For networks of small organisations (SMEs) these challenges are amplified, as resources are scarcer and high entry costs are prohibitive. Challenges like these are not unique for creative business networks. They occur, usually to a lesser extent, in projects inside organisations as well. Dynamic creative business networks are difficult to support with traditional system development because of the transient and situated nature of each virtual enterprise. For one organisation, the number of potential partners is huge, and with each partner they are perhaps involved in only a few projects. 
Consequently, integrating the infrastructures through traditional systems development practices is not economically viable. Standardisation is often proposed in these cases, but standards require that the domain is well understood and established. This is seldom the case for knowledge intensive work processes. Consequently, we need a more flexible approach; one that allows the solution to emerge as the co-operation matures.

\section{ACTIVE MODELS}

Whereas in knowledge-based systems, the operational logic is stored in a database rather than programmed in software, reflective systems expose representations of their own logic to their users, and allow modification of this logic [6]. Active models combine these features with user interaction through graphical models $[5,16]$. Several recent trends in information systems engineering illustrate the increasing importance of active models. Workflow [4, 14], information sharing [22], document classification and retrieval [3], product data management [8], and co-operation support [11] are areas where active models have been applied.

Models are generally defined as explicit representations of some portions of reality as perceived by some actor. In information systems development, conceptual models have long been used to analyse the problem domain, capture and structure user requirements and design the system. Active models, on the other hand, are also actively used during the operation of the system. What does it mean that a model is active? First of all, the representation must be available to the users of the information system at runtime. Second, the model must influence the behaviour of the underlying computerised system. Third, the model must be dynamic, users must be supported in changing the model to fit their local reality, enabling tailoring of the system's behaviour. Let us illustrate this with the example of workflow management systems (WMS) $[4,28]$. Such systems support the co-ordination of work in business processes. The active models in WMS are process models. Process models are especially important because they represent ways of working, organisational routines and theories of action [2]. They reflect the tasks that are part of the process, their interdependencies and the resources that are applied to perform them. Resources include personnel, information and tools. All workflow systems include an enactment service that interprets the model as work progresses, filling the users to-do-lists with new tasks when the model says they are ready to be performed. Most workflow systems also include a process definition component that enables users to create and update models. In static workflow, process models are built by experts and not allowed to change while the process is being executed. This solution has been 
experienced to only work for well-understood, routine processes. Consequently, adaptive workflow is an important research area $[1,4,14$, $24,28]$. Here models are allowed to change, and change will affect ongoing processes. In emergent workflow [14], modelling is viewed as an integral part of the work, performed by the process participants, as they are the only actors who have sufficient knowledge of the process. The focus is unique cases, especially knowledge intensive projects. WMS thus illustrate the primary characteristics of active models:

- Models are available at runtime, in the process definition component,

- Models influence system behaviour, through the enactment component,

- Models are dynamic, to a varying degree in production, adaptive and emergent workflow.

- Models increase flexibility by letting users define control sequences that were previously programmed in software.

\section{THE EXTERNAL INFRASTRUCTURE}

It is the hypotheses of the EXTERNAL project [7] that the active model approach is suitable for supporting digitally enabled creative business networks. The term 'extended enterprise' refers here to such model-integrated virtual enterprises. We are currently integrating the functionality and concepts of a number of enterprise and process modelling and enactment tools to form a suitable infrastructure for our case studies, and we have tested initial versions of this infrastructure. We are currently undergoing a separate evaluation task to investigate the pros and cons of the approach, and have a better basis for the development of the next version. This section gives a brief description of the current EXTERNAL infrastructure and its utilisation of active models, which are the core means of integration. We will also present the next version of the infrastructure that we are currently working on, and which will be ready for use in the end of the summer of 2002.

The current infrastructure integrates four tools and prototypes developed or in use by the project partners prior to the project:

- METIS [18], a general purpose enterprise modelling and visualisation tool,

- XCHIPS [11], a co-operative hypermedia tool integrated with process and communication support,

- SIMVISION (earlier Vite) [17], a project simulator that analyses resource allocation, highlighting bottlenecks and actor backlogs. 
- WORKWARE [14], an emergent workflow management system with a web-browser-interface with to-do-lists, process-trees, document sharing, process enactment, awareness mechanisms and email integration.

A portal solution enables a unified access to the different tools. Links to a traditional workflow automation system called FrameSolutions is currently also developed. Also more traditional off-the-shelf functionality is provided by integrating the use of tools such as NetMeeting and Webex.

Together the tools of the EXTERNAL infrastructure offer a comprehensive suite of functionality for creating, maintaining, and utilising shared active nodels of the extended enterprise (EE) as illustrated in Figure 1.

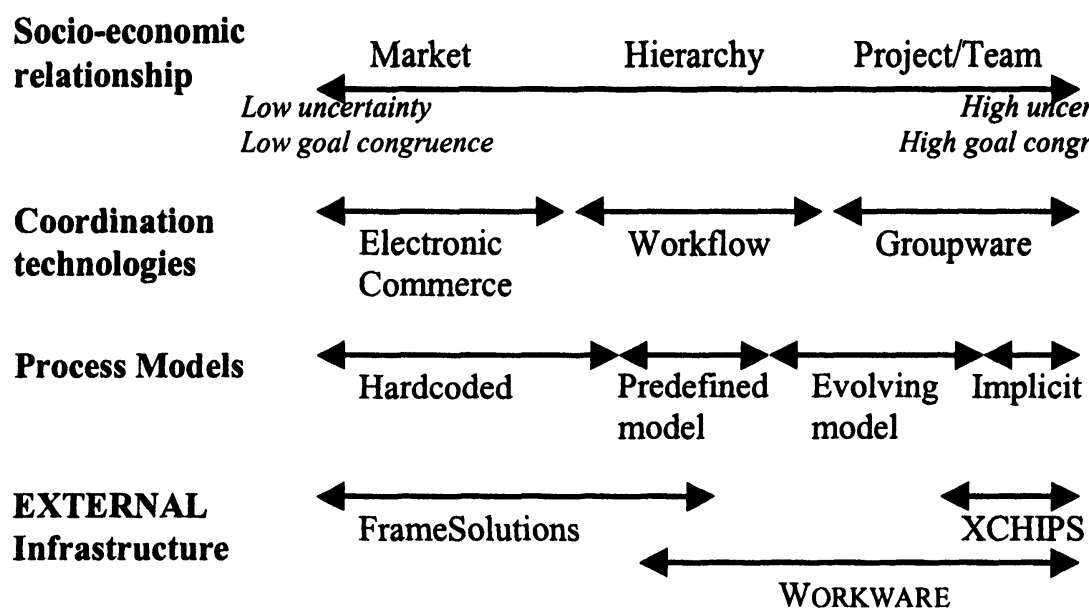

Figure 1. Coordination technologies for different forms of collaboration

Different parts of the implemented, integrated modelling languages are applicable for different types of process modelling. To integrate process technologies from the enterprise perspective of generic business process types to the emergent workflow perspectives at the instance level, we have identified 4 layers of process knowledge representation, from general process logic to actual, situated work performance. Process modelling occurs at several levels concurrently, and may start at any level.

- Layer 1 - Describe Process Logic: At this layer, one identify the constituent activities of generic, repetitive processes and the logical dependencies between these activities. A process model at this layer should be transferable across time and space to a mixture of execution environments. Examples of process logic are conceptual value chains and normative descriptions of "best practices". 
- Layer 2 - Engineer Activities: Here process models are expanded and elaborated to facilitate business solutions. Elaboration includes concretisation, decomposition, and specialisation. Integration with local execution environment (particular extended enterprises) is achieved e.g. by describing resources required for actual performance.

- Layer 3 - Manage Work: The more abstract layers of process logic and of activity description provide constraints, but also useful resources (in the form of process templates) to the planning and performance of each extended enterprise process. When modelling at layer 3, detailed decisions are taken regarding the performance of work in the actual work environment with its organisational, information, and tool resources; the scope is narrowed down to actual process instances (e.g. a specific project). Concrete resources increasingly are intertwined in the model, leading to the introduction of more dependencies. Management of activities may be said to consist of detailed planning, co-ordination and preparation for resource allocation.

- Layer 4-Perform Work: Covers the actual execution of tasks according to the determined granularity of work breakdown, which in practice is coupled to issues of empowerment and decentralisation. When a group or person performs the task, whether to supply a further decomposition may be left to their discretion, or alternative candidate decompositions might be provided as advisory resources. At this layer resources are utilised or consumed, in an exclusive or shared manner.

Process knowledge management: This activity can be defined as the collection of processes necessary for innovation, dissemination, and exploitation of knowledge in a co-operating ensemble where knowledge seekers are linked to knowledge sources and a shared knowledge base is cultivated. Process knowledge management is active at all layers of this model. In particular, models of past process instances - typically situated at the lower layers - can be subject to a harvesting process in order to update templates available at the higher layers. Model templates and fragments from participating organisations may be used as components in the active knowledge models of a particular extended enterprise. Likewise, the shared knowledge base will be used to support learning at the individual, team and organisational level.

The models are managed by a shared repository residing on a web server. For the representation and interchange of active models, an XML DTD is defined. METIS is used for building and visualising rich, up-to-date models of the joint project, fostering common understanding among the participants and enabling them to plan their co-operation. XCHIPS allow for synchronous collaborative modelling sessions and also same-time collaboration in the context of particular tasks. SIMVISION and WORKWARE activate the 
models in different ways. SIMVISION simulates the expected future course of events in the enterprise, given the current work plans (process models), personnel allocation and organisation. WORKWARE utilise the active models to build contextual and customisable user interfaces that give users access to the relevant information, tools, and communication channels for each particular task. In other words, XCHIPS provides contextual work support for focussed, real-time collaboration, whereas WORKWARE does the same for asynchronous collaboration.

The overall infrastructure is best described as consisting of four layers. These layers are identified as:

- Layer 1, the ICT layer: - defining and describing the technology platform, the software architectures, tools, software components and capabilities, connectivity and communications.

- Layer 2, the Knowledge Representation layer: - defining and describing modelling languages, developing, sharing and managing the contents of model and meta-model repositories.

- Layer 3, the Model and Work Management layer: - modelling the customer solution, adapting engineering processes, and implementing work processes, executing and managing models.

- Layer 4, the Work Performance layer; - implementing customer solutions, generating work environments as personalised and contextsensitive views and GUI's (worktops) available through portals, and performing work with life-cycle management control.

\section{MEETING THE CHALLENGES OF DIGITALLY ENABLED CREATIVE BUSINESS NETWORKS WITH ACTIVE MODELS}

This section briefly outlines how the active models approach and the EXTERNAL infrastructure can meet the challenges of supporting digitally enabled creative business networks, as listed above.

- The EE models capture a rich set of relationships between the organisations, people, processes and resources of the virtual enterprise. Through analysis and activation (e.g. WORKWARE's enactment), the models become applied as sources of knowledge, providing the basis for knowledge exchange.

- Local modifications to match the needs of a particular enterprise are supported by instance modelling [14]. This limit the scope of a change to the local situation, removing much of the complexity that has prevented 
modelling by end users at the more generic level. It is also a prerequisite for establishing an immediate connection between the domain and the active model, enabling learning and knowledge management anchored in practice, as discussed above. For externalisation and user participation, it is important to keep the core modelling languages simple. Increased expressiveness and detailed semantics may be harmful, especially if the model is expected to change and be refined as the work progresses.

- Planning, the joint construction on an active enterprise model by the participants, is supported by the modelling tools, especially the real-time collaborative modelling of XCHIPS.

- Communication is supported through the infrastructure tools, and the terminology of the modelling language. The joint modelling of the enterprise facilitates common understanding, and the extensibility of the EE modelling language enables local shared understanding to be expressed and utilised.

- Model-driven access control mechanisms can utilise the model of organisations' and persons' roles in the tasks of the joint enterprise to deduce whom should have access to what, subject to customisable policies.

- Flexibility is ensured through interactive model interpretation, combining the capabilities of the system to automate predefined parts and the users to handle incompletely specified parts of the model [14]. This enable the total system to handle models with varying, user-controllable degrees of specificity, where structure can emerge as the users' understanding of the domain increases.

- Model interpretation is contextual. This implies that the meaning of a model element should depend on the current situation. In WORKWARE, the meaning of process flows depends on the states of the tasks it relates. If the source task is completed before the target is started, then the enactment engine activates the target. But if both are active in parallel, the awareness server takes control and uses the relationship as a channel for awareness mediation. Interactive and contextual interpretation of model elements enables simpler modelling languages.

- The system architecture can benefit from integrating multiple modelactivator components. In addition to workflow enactment, WORKWARE uses the relationships between tasks and documents for information management and the process flow structure for awareness mediation. The enactment engine and the awareness server both activate the process model to support co-ordination. The engine activates the predefined flows of work, and involves the users when incompleteness in the models disables automatic reasoning. The awareness server informs people about the actions on tasks that are related to their tasks, enabling co-ordination 
through mutual adjustment. This is especially important in models with little predefined structure.

- Flexibility with respect to the participation of organisations in the virtual enterprise, rapid formation, and integration of capabilities, is handled by updating the organisation sub-model. This will automatically influence the access control and the rest of the infrastructure.

In all, active models seem to be a promising candidate for addressing a number of the challenges that we have identified. Further work is needed both to refine the rudimentary support that the EXTERNAL infrastructure currently offers in some areas as outlined above, and to explore how the many aspects that need to be represented in a model can be integrated without complicating the modelling language and thus limiting its practical usability.

\subsection{Case studies and theoretical grounding}

More work is needed to validate the usability of the active model approach. In EXTERNAL three different case studies are currently underway, providing a representative selection of knowledge intensive virtual enterprises. One consists of two business consulting firms with complementary expertise who collaborate with their joint customers. The second is a network of SMEs, collaborating to provide IT services. The third is an international research project. Major parts of our infrastructure are also being used to support several ongoing, industrial projects. Currently, we are evaluating the effects of using the infrastructure. The evaluation work aims at investigating the quality of EXTERNAL in terms of supporting work in extended enterprises. More specifically the evaluation whether the implementation of EXTERNAL, into the 3 different extended enterprises currently using standard IT support-tools, cause a traceable simplification in the way these can be operated. Our hypothesis is that the ease with which an EE can be operated increases with the implementation of the EXTERNAL solutions.

In order to evaluate the quality of EXTERNAL we have followed a quasi-experimental strategy as we measure how participants of the 3 separate EE's in the case studies perceive their working environment, before and after EXTERNAL is implemented. A quasi experiment resembles a classical experiment in the social sciences, since specific attitudes of a group of people are measured before and after the group is given a stimulus believed to impact on these attitudes. However, in a classical experiment the attitudes of a control group (that is not given the stimulus) are compared to the group that is subject to the stimulus. In addition, both the experimental group and the control group are selected by randomisation. This evaluation does not 
encompass a control group and the respondents are not selected by randomisation, hence it falls into the category of quasi experiments. Since this research strategy only provides time-series data for the experiment group (pre and post-test), the professional judgement of the researcher (which in this case is external to the actual project) becomes more important than statistical measures when conclusions about the evaluation are to be made.

In order to provide the best possible basis for such judgement, measurements has been performed by way of a structured questionnaire submitted to all EE participants before and after the implementation of the EXTERNAL infrastructure. In addition, semi-structured interviews have been conducted with selected participants. This is done since we are lacking a control group. It is a case study, hence we can not perform selection by randomisation. The use of interviews is performed to provide more depth and to unveil areas of improvement. A detailed discussion of the results of these studies can be found in [19] which builds up on our preliminary results reported above. The interactions between users and researchers in the project ensure an ongoing practical validation. In addition, our work has a strong theoretical grounding in organisational and social sciences $[4,12]$, as well as in new conceptions of information systems modelling $[16,27]$.

\subsection{Related work}

The EXTERNAL infrastructure combines a number of mechanisms that use active models for customising and personalising the information systems, e.g. simulation, workflow enactment, awareness, user interface layout and navigation design. Model-based multilevel access control is a further objective for our extended enterprise infrastructure. With respect to supporting dynamically networked organisations, most B2B E-business frameworks [25] focus on information exchange and business transactions. They lack support for the dynamic and knowledge-intensive parts of interorganisational processes. Enterprise ontologies have been proposed as a solution to the communication problems arising from different interpretative frameworks in different organisations [9]. Unlike interactive enactment, this approach is based on conventional notions, where the model interpretation is fully automated and without any user involvement. This leads to static models where activation is based on inferences made by the system only. Active models are also directed towards ongoing modelling, model interpretation, and activation by the end users. Our objective is not to maximise the expressiveness and inference power of the computerised information system, but to increase the effectiveness of the whole information system (including the users). Following some of the rationale 
behind active models, systems for maintaining dynamic ontologies have recently been developed.

Workflow management systems have also been proposed as a solution for interorganisational collaboration [1, 24]. The focus of EXTERNAL on knowledge intensive processes requires a degree of flexibility not enabled by static, or even adaptive, workflow systems. Flexible workflow is an important research area, to which our approach contributes with a focus on instance modifications by process participants, and with richer co-ordination support based on interactive enactment [14]. Another popular solution for cross-enterprise integration is middleware frameworks like OMG's CORBA [23]. The recent shift in the focus of OMG to modelling (UML and MDA), standardisation of meta-object integration (MOF), business object frameworks and workflow management facilities indicate an interest in model-driven enterprise integration also from the more technical side. Whether these software engineering approaches are directly transferable to active modelling remains to be investigated thoroughly.

\section{CONCLUSIONS AND FURTHER WORK}

This paper has pointed to active models, created and used during the operation of information systems, as a promising approach to increasing the flexibility of information systems in general, and cross-organisational information systems in particular. The work of the EXTERNAL project on active models as a means to support the establishment and operation of digitally enabled creative business networks has served as a practical case. We have briefly outlined some of the many ways in which the tools of the EXTERNAL infrastructure utilise active enterprise and process models to facilitate knowledge creation, sharing and collaboration. Further experimentation with this approach as a basis for improving modelling languages, infrastructure, and the modelling methodology is the major challenge for the remainder of this project. Our ongoing research also targets the problem of generalising local modification and innovation, packaging them in a manner suitable for reuse [13, 15], thus enabling knowledge management and learning from practice.

\section{REFERENCES}

1. Alonso, G., Fiedler, U., Hagen, C., Lazcano, A., Schuldt, H. and Weiler, N. WISE:

Business to Business E-Commerce, 9th International Workshop on Research Issues on Data Engineering (RIDE-VE'99), Sydney, Australia, 1999. 
2. Argyris, C. and Schön, D. Organizational Learning: A Theory of Action Perspective. Reading, MA, USA: Addison Wesley, 1978.

3. Brasethvik, T. and Gulla, J. A. Semantically accessing documents using conceptual model descriptions, Workshop on Web and Conceptual Modelling, ER 99, Paris, France, 1999.

4. Carlsen, S. Conceptual Modeling and Composition of Flexible Workflow Models, PhDthesis 1997, NTNU - Norwegian University of Science and Technology, Trondheim, Norway.

5. Carlsen, S., Johnsen, S. G., Jørgensen, H. D., Coll, G. J., Mæhle, A., Carlsen, A. and Hatling, M. Knowledge re-activation mediated through knowledge carriers, MICT'99, Copenhagen, Denmark, 1999.

6. Dourish, P. Developing a Reflective Model of Collaborative Systems, ACM Transactions on Computer-Human Interaction, vol. 2, no. 1, 1995.

7. EXTERNAL EXTERNAL - Extended Enterprise Resources, Networks And Learning, EU Project, IST-1999-10091, New Methods of Work and Electronic Commerce, Dynamic Networked Organisations. Partners: DNV, GMD-IPSI, Zeus E.E.I.G., METIS, SINTEF Telecom and Informatics, 2000-2002.

8. Farshchian, B. A. Gossip: An Awareness Engine for Increasing Product Awareness in Distributed Development Projects, in Advanced Information Systems Engineering Proceedings of CAiSE 2000, vol. LNCS 1789, B. Wangler and L. Bergman, Eds. Stockholm, Sweden: Springer, 2000.

9. Fox, M. S. and Gruninger, M. On Ontologies and Enterprise Modelling, International Conference on Enterprise Integration Modelling Technology 97, 1997.

10. Hruby, P. Structuring Specification of Business Systems with UML (with an Emphasis on Workflow Systems), OOPSLA'98 Business Object Workshop IV, 1998.

11. Haake, J. M. and Wang, W. Flexible Support for Business Processes: Extending Cooperative Hypermedia with Process Support, GROUP '97, Phoenix, Arizona USA, 1997.

12. Jørgensen, H. D. Active Models in Information Systems, NTNU, Trondheim, Norway, "Organisation, Technology and Change" Course 2000.

13. Jørgensen, H. D. Software Process Model Reuse and Learning, Process Support for Distributed Team-based Software Development (PDTSD'00) as part of the 4th World Multiconference on Systemics, Cybernetics and Informatics (SCI 2000), Orlando, Florida, 2000.

14. Jørgensen, H. D. and Carlsen, S. Emergent Workflow: Integrated Planning and Performance of Process Instances, Workflow Management '99, Münster, Germany, 1999.

15. Jørgensen, H. D. and Carlsen, S. Writings in Process Knowledge Management, SINTEF Telecom and Informatics, Oslo, Norway STF40 A00011, 2000.

16. Jørgensen, H. D. and Krogstie, J. Active Models for Co-operative Information Systems, NIK'01, Tromsø, Norway November 2001.

17. Kuntz, J. C., Christiansen, T. R., Cohen, G. P., Jin, Y. and Levitt, R. E. The Virtual Design Team: A Computational Simulation Model of Project Organizations, Communications of the ACM, vol. 41, no. 11, 1998.

18. Lillehagen, F. Visual Extended Enterprise Engineering Embedding Knowledge Management, Systems Engineering and Work Execution, IEMC '99, IFIP International Enterprise Modelling Conference, Verdal, Norway, 1999.

19. Lillehagen, F., Krogstie, J. Jørgensen, H., Hildrum, J. Active Knowledge Models for supporting eWork and eBusiness. To be presented at ICE'-2002, June 2002.

20. Loos, P. and Allweyer, T. Process Orientation and Object-Orientation - An Approach for Integrating UML with Event-Driven Process Chains (EPC), University of Saarland, Saarbrücken, Germany 1998.

21. Marshall, C. Enterprise Modeling with UML: Addison-Wesley, 1999.

22. Natvig, M. K. and Ohren, O. Modelling shared information spaces (SIS), GROUP '99, Phoenix, Arizona USA, 1999.

23. OMG CORBA/IIOP Specification v. 2.4.1 : OMG - Object Management Group, 2000. 
24. Reichert, M., Bauer, J. and Dadam, P. Enterprise-Wide and Cross-Enterprise WorkflowManagement: Challenge, and Research Issues for Adaptive Workflows, Enterprise-wide and Cross-enterprise Workflow Management, Paderborn, Germany, 1999.

25. Shim, S. S. Y., Pendyala, V. S., Sundaram, M. and Gao, J. Z. Business-to-Business ECommerce Frameworks, IEEE Computer, vol. 33, no. 10, 2000.

26. Suchman, L. Plans and Situated Actions. New York: Cambridge University Press, 1987.

27. Wegner, P. and Goldin, D. Interaction as a Framework for Modeling, in Conceptual Modeling. Current Issues and Future Directions, Lecture Notes in Computer Science 1565, P. P. Chen, J. Akoka, H. Kangassalo, and B. Thalheim, Eds. Berlin, Germany: Springer, 1999.

28. Aalst, W. v. d., Desel, J. and Oberweis, A. Business Process Management. Berlin, Germany: LNCS 1806, Springer, 2000. 